\title{
The Photovoltaic Response of Intrinsic Organic Semiconductor Single Crystals
}

\author{
M. Campione*, D. Braga, L. Raimondo, M. Moret, A. Sassella, S. Binetti and M. Acciarri \\ Department of Materials Science, Università degli Studi di Milano Bicocca, Via Cozzi 53, I-20125 Milan, Italy
}

\begin{abstract}
Rubrene, perylene, $\alpha$-quaterthiophene, and tetracene single crystals were grown and placed between indiumtin-oxide and Al electrodes; the current-voltage characteristics of the so-fabricated two-terminal organic devices were measured at ambient conditions under solar simulator illumination. While rubrene single crystals do not exhibit any photovoltaic effect, open-circuit voltages reach values up to $1.8 \mathrm{~V}$ in devices based on perylene, $\alpha$-quaterthiophene, and tetracene single crystals.
\end{abstract}

Keywords: Organic semiconductors, single crystals, organic solar cells.

\section{INTRODUCTION}

Organic semiconductors based on low molecular-weight molecules such as porphyrins, phthalocyanines, oligothienyls, oligophenyls, and oligocenes, have been extensively employed as active layers in simple photovoltaic (PV) devices [1]. In these kinds of devices the semiconducting layer is usually present as a polycrystalline thin film phase comprising two different materials, one acting as electron donor and the other as electron acceptor, assembled in a double layer or bulk heterojunction. Exploiting thin film architectures is a natural choice since organic materials display an intrinsic high resistivity and relatively low exciton diffusion length (a few tens of nanometers). Only one example in the literature is reported where single crystals of tetracene with thickness down to $200 \mathrm{~nm}$ are employed as donor layers in double layer heterojunction solar cells with a film of $\mathrm{C}_{60}$ as acceptor layer, reaching a power conversion efficiency of $0.34 \%$ [2].

The propensity of organic semiconductors based on small molecules to give rise to crystalline phases is an advantageous characteristic (in comparison to polymeric semiconductors, which form solid phases with large amorphous fractions) since the 3D periodicity enhances the exciton diffusion length and the charge mobility. On the other hand, the exciton binding energy is relatively high in molecular crystals, making the separation of electron-hole pairs fairly difficult [3]. In this framework, in order to get useful guidelines to the choice of suitable materials for the fabrication of efficient small-molecule organic solar cells, we analyzed the intrinsic PV response of the organic semiconductors through the investigation of the best-defined model systems, i.e. single crystals. In this letter, we study the PV response of pure single crystals of well known organic semiconductors, namely rubrene (RUB, $\mathrm{C}_{42} \mathrm{H}_{28}$ ), perylene (PEL, $\mathrm{C}_{20} \mathrm{H}_{12}$ ), $\alpha$ quaterthiophene $\left(\alpha-4 \mathrm{~T}, \mathrm{C}_{16} \mathrm{H}_{10} \mathrm{~S}_{4}\right)$, and tetracene (TEN, $\mathrm{C}_{18} \mathrm{H}_{12}$ ), placed between indium-tin-oxide (ITO) covered

*Address correspondence to this author at the Department of Geological Sciences and Geotechnologies, Università degli Studi di Milano Bicocca, Piazza della Scienza 4, I-20126 Milano, Italy; Tel: +39 6448 2089; Fax: +39 6448 2073; E-mail: marcello.campione@unimib.it glass plate and Al electrodes (Fig. (1)). Previous studies bypassed this fundamental step, probably due to the difficulties in growing and handling high quality single crystals, and to the problems related to the metallization of their surfaces.

RUB has been recently used in its thin film phase for fabricating organic dual devices (electro-luminescent and PV) with $\mathrm{C}_{60}$ as acceptor layer, reaching a power conversion efficiency of 3\% and a photovoltage of $1 \mathrm{~V}$ [4]. On the contrary, PEL and $\alpha-4 T$, even though their semiconducting behavior has been demonstrated in several experiments $[5,6]$, have never been used for fabricating PV devices. We will show that each semiconductor presents distinctive characteristics in terms of short-circuit current density, open-circuit photovoltage, and power-conversion efficiency. In particular, while RUB single crystals does not show any detectable PV response (though exhibiting the highest conductivity), PEL, $\alpha-4 T$, and TEN single crystals give rise to an apparent PV behavior, reaching open-circuit voltages up to $1.8 \mathrm{~V}$.

\section{GROWTH AND OPTICAL CHARACTERIZATION OF SINGLE CRYSTALS}

Commercial RUB, PEL, $\alpha-4$ T, and TEN powders were purified by several sublimation steps before use. Single crystals of RUB, TEN, and PEL were grown from the vapor phase following the procedures reported in Ref. [7], whereas $\alpha-4 \mathrm{~T}$ single crystals were grown from anisole solution by the floating-drop method [8]. The samples were placed without any intermediate layer on a quartz plate. Single crystals are obtained as thin flakes (thickness up to $1 \mu \mathrm{m}$ ), some millimeters wide exposing a clean, molecularly flat surface, which does not require cleavage before use. The low aspect-ratio of such crystals is due to the high anisotropy of the intermolecular forces. The $\pi$-conjugate system drives the formation of a layered structure with low energy surfaces separating adjacent compact layers (see Fig. (1)). These low-energy surfaces are easily identified in the crystalline sample since they are the widest ones. RUB is known to crystallize in three different polymorphs: triclinic, monoclinic, and orthorhombic. Vapor phase methods produce the orthorhombic polymorph with (100) as the widest (contact) surface [9]. The triclinic and monoclinic polymorphs are obtained by solution 


\section{RUBRENE}
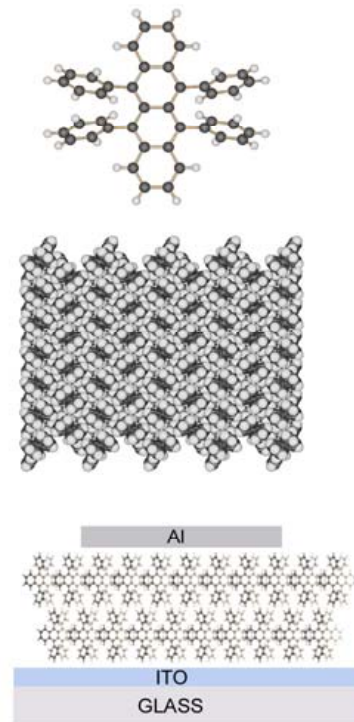

PERYLENE
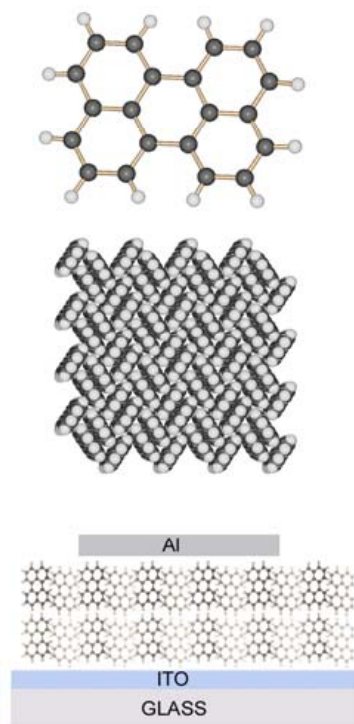

$\alpha$-QUATERTHIOPHENE
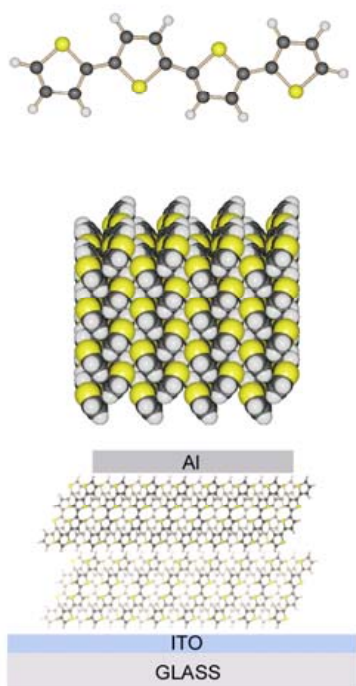
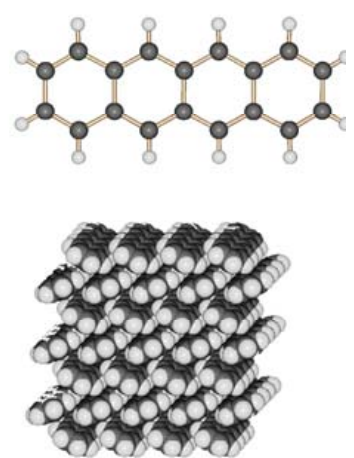

TETRACENE

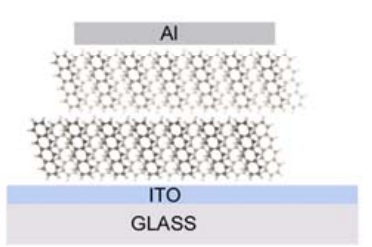

Fig. (1). Molecular structures (top), contact surface of the grown single crystals (center), and device configuration (bottom) of RUB (orthorhombic polymorph), PEL (monoclinic $\alpha$-polymorph), $\alpha$-4T (monoclinic low-temperature polymorph), and TEN (triclinic polymorph). Grey: C-atoms, white: H-atoms, yellow: S-atoms.

methods [10]. Two monoclinic polymorphs of PEL are known: the $\alpha$-polymorph, appearing as a rectangular, tabular, yellow crystal, and the $\beta$-polymorph, appearing as a hexagonal, greenish-yellow prism [11]. Vapor phase methods produced the $\alpha$-polymorph of PEL with (001) as the widest (contact) surface [12]. Depending on the source temperature, vapour phase methods have been demonstrated to produce $\alpha$ 4T crystals with two different monoclinic phases: the low temperature polymorph for temperatures $\leq 140^{\circ} \mathrm{C}$ and the high temperature polymorph for temperatures $\geq 160{ }^{\circ} \mathrm{C}$ [13]. Room temperature solution methods produce the low temperature polymorph of $\alpha-4 \mathrm{~T}$, with (001) as the widest (contact) surface [8]. Finally, only a triclinic phase of TEN is known [14], with (001) as the widest (contact) surface [15]. A sketch of the molecules and of the surfaces of the three materials is reported in Fig. (1).

In order to corroborate the phase and orientation assigned above and to evaluate the overlap between the solar irradiance and optical response of these four organic semiconductor crystals, optical absorption spectra have been performed at normal incidence on the contact face of small crystals (few hundreds nanometers thick) in the visible spectral range with a Perkin-Elmer Lambda900 spectrophotometer. Fig. (2) reports the absorption spectra collected under polarized light for making evidence of the typical optical anisotropy of such crystals which is in turn related to their highly anisotropic structures. Incident light has been polarized along orthogonal crystal directions, chosen as those characterizing the direction of polarization of the lowest energy exciton transitions (mainly of Frenkel origin). The main peaks detected show the spectral position and lineshape expected for the orthorhombic polymorph of RUB(100), for the $\alpha$-polymorph of PEL(001), for the low temperature polymorph of $\alpha-4 T(001)$, and for the triclinic polymorph of TEN(001), in full agreement with all the available data in literature [12, 16-18].

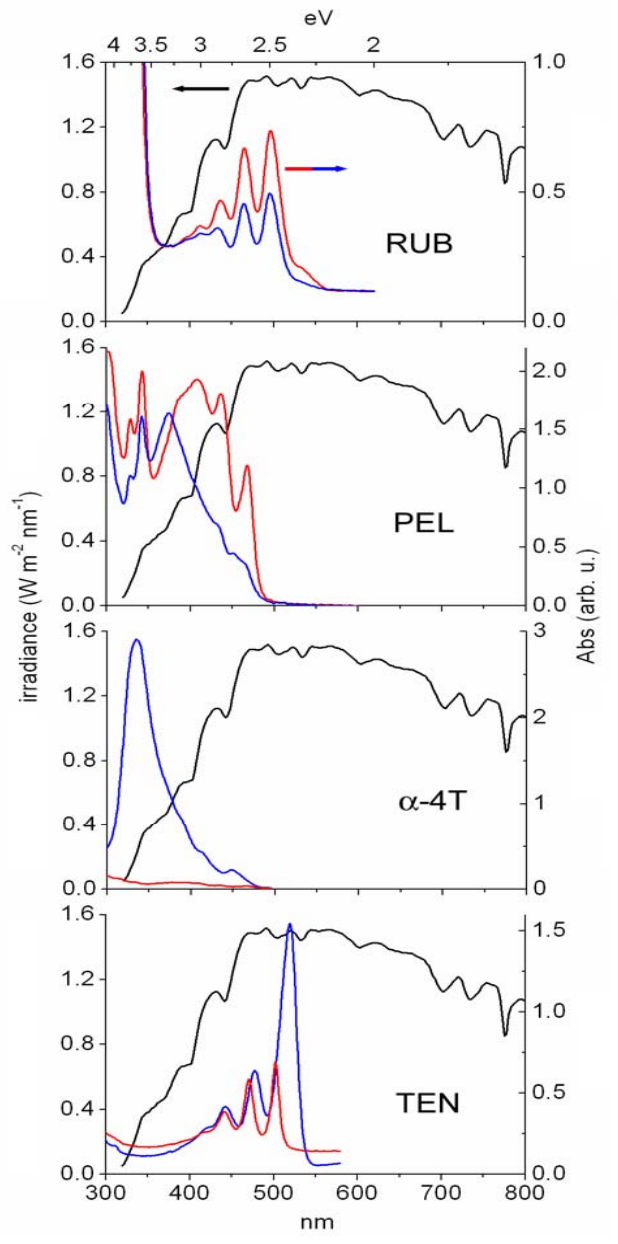

Fig. (2). Comparison of the solar irradiance at sea-level (black lines) and the normal incidence absorbance spectra of RUB, $\alpha-4 T$, PEL, and TEN single crystals. Red (blue) lines: electric field direction of the incoming light parallel (perpendicular) to [010]. 


\section{DEVICE ASSEMBLY AND ELECTRICAL CHARAC- TERIZATION}

Single crystals of RUB, PEL, $\alpha-4 T$, and TEN, typically $20 \div 40 \mathrm{~mm}^{2}$ wide and $1 \mu \mathrm{m}$ thick, were placed without any intermediate layer on a boron-silicate glass plate $\left(1 \mathrm{~cm}^{2}\right)$ covered with an indium tin oxide (ITO, 30-60 $\Omega / \square$, surface roughness $3.5 \mathrm{~nm}(\mathrm{rms})$ over a $1 \mu \mathrm{m}^{2}$ area) layer (Fig. (1)). The metallization carried out by thermal evaporation on PEL and $\alpha-4 \mathrm{~T}$ is not a suitable method because of the consequent surface damage. When this method is used, introduction of structural defects is an inevitable and well-known problem [19] and metal penetration into the volume of the organic active layer is often observed [20,21]. Indeed, as can be observed in the atomic force microscopy (AFM) images reported in Fig. (3) [22], the surface of these crystals undergoes dramatic changes at room temperature when kept in ultra-high vacuum conditions $\left(1 \times 10^{-9} \mathrm{mbar}\right)$. For the PEL crystal Fig. (3a and b), vacuum is observed to induce sublimation, since a terrace appearing at the top-right in Fig. (3a) completely disappears in Fig. (3b), and the surface roughness increases due to the high density of residual islands. Some voids also form, as can be observed in the bottom-left and top regions of Fig. (3b). A similar behavior is observed for $\alpha-4 \mathrm{~T}$ crystals; Fig. (3c and $\mathbf{d}$ ) show the evolution of a step-edge, which is observed to move backwards due to the onset of sublimation; furthermore, sublimation of a second layer is observed, giving rise to a depleted region having the same profile of the original step-edge. As an alternative option, top contacts were fabricated using an $\mathrm{Al}$ tape [23] (4 $\mathrm{mm}^{2}$ ca.), assuring the direct contact between the semiconductor and the metal and avoiding any mechanical damage. The ultimate assemblies were self-standing.
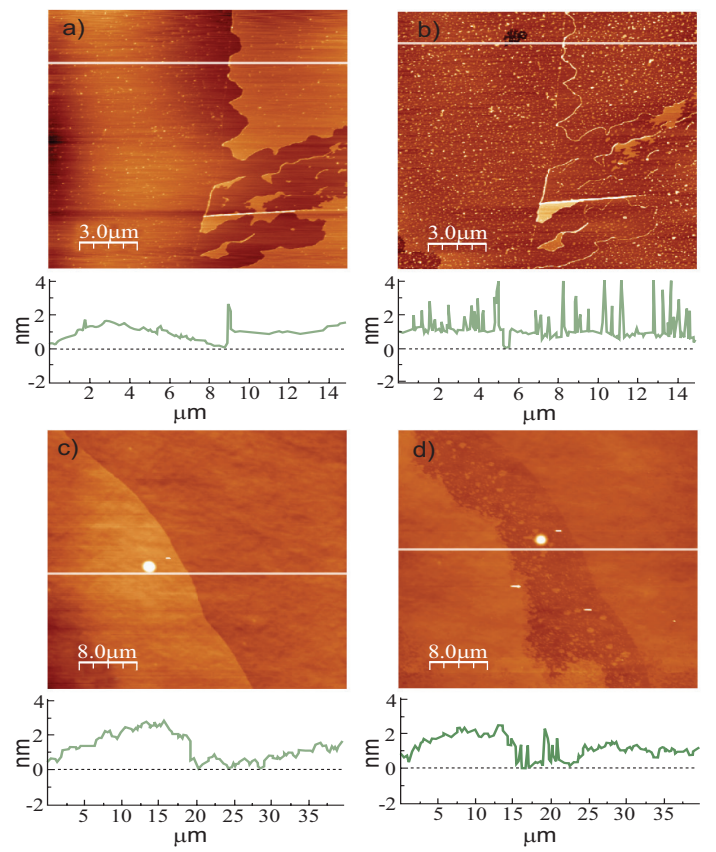

Fig. (3). AFM images (tapping mode: cantilever force constant 40 $\mathrm{N} / \mathrm{m}$, resonance frequency $250 \mathrm{kHz}$ ) collected over the same region of the surface of PEL and $\alpha-4$ T crystals before ( $a$ and c) and after ( $b$ and d) permanence at room temperature in ultra-high vacuum for 60 h. Roughness (root mean square) values are a) $0.68 \mathrm{~nm}, \mathrm{~b}$ ) $0.70 \mathrm{~nm}$, c) $0.80 \mathrm{~nm}, \mathrm{~d}) 1.3 \mathrm{~nm}$. The cross-sectional profiles below each couple of images are taken along the white lines.
The devices were characterized by collecting current/voltage characteristics with a Keithley 24001 A sourcemeter, illuminating the ITO side in air with a ThermoOriel Solar Simulator with power density of $89 \mathrm{~mW} / \mathrm{cm}^{2}$.

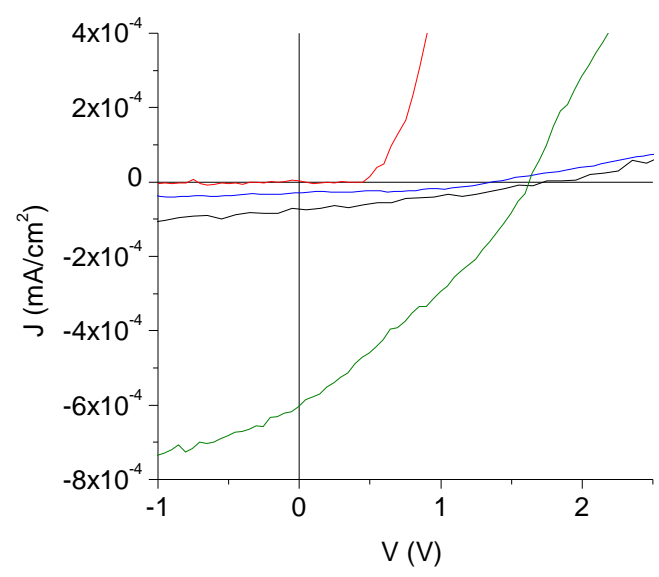

Fig. (4.) $J-V$ characteristics under AM1.5 illumination of single crystal solar cells fabricated with ITO and Al electrodes. red: RUB, blue: PEL, Black: $\alpha-4 \mathrm{~T}$, green: TEN.

Fig. (4) shows the $J-V$ characteristics under AM1.5 illumination of RUB, PEL, $\alpha-4 \mathrm{~T}$, and TEN cells. The PV parameters extracted from the curves in Fig. (4) are reported in Table 1. Among the considered materials, only RUB crystals exhibits a photocurrent lower than $10^{-10} \mathrm{~A}$, then near the detection limit of the electrometer. For the other materials, the onset of a PV effect is apparent, though photocurrents are apparently low; this is due to the high resistance of the organic layers and, especially for PEL and $\alpha-4 T$, the small overlap of the absorbance and solar irradiation spectra (Fig. (2)). Moreover, it must be noted that, with the device configuration used (see Fig. (1)), current flow is highly unfavored because charge carriers must follow a path orthogonal to the molecular layers, along which the overlap of $\pi$ orbitals, responsible for charge transfer from a molecule to another, is negligible. Interestingly, open-circuit voltages $\left(V_{o c}\right)$ reach relatively high values if compared with those of donor-acceptor hetero-junctions. Indeed, the presence of a single active layer prevents the energy loss associated with the electron transfer at the donor-acceptor interface from the LUMO level of the donor to that of the acceptor; then, $V_{o c}$ values overcome $1 \mathrm{~V}$, reflecting the high exciton energy of the single organic layer. Note, e.g., that in TEN single crystal/C60 bi-layer heterojunction solar cells, the measured $V_{o c}$ was $0.57 \mathrm{~V}$ [2], to be compared with $1.6 \mathrm{~V}$ (see Table 1) obtained with our TEN single crystal device. Since PEL, $\alpha$ 4T, and TEN single crystal cells are hole-only devices, charge separation of the light-induced exciton likely occurs at the crystal/Al interface. As exciton diffusion lengths are of the order of some tens of nanometers, whereas the crystal thickness is hardly lower than $200 \mathrm{~nm}$, only a (small) fraction of the incident light can contribute to charge generation. All these effects are responsible for the modest estimated power conversion efficiencies; nonetheless, it must be noted that they are of the same order of those obtained with thick bulk heterojunction solar cells [24]; furthermore, the efficiency of our TEN single layer solar cell reaches that obtained with TEN single crystal/C60 bi-layer heterojunction 
Table 1. Photovoltaic Response of Organic Single Crystal Solar Cells. Short-Circuit Current Density $\left(J_{s c}\right)$, Open-Circuit Voltage $\left(V_{o c}\right)$, Fill-Factor $(F F)$, and Power Conversion Efficiency $(\eta)$ of Solar Cells Fabricated with Single Crystals of RUB, PEL, $\alpha-4 T$, and TEN. Uncertainties Associated with the Measured Quantities are of the Order of 5\%. Optical Absorption Edges $\left(E_{G}\right)$ are Reported in the Last Column.

\begin{tabular}{|c|c|c|c|c|c|}
\hline & $\mathrm{J}_{\mathrm{sc}}\left(10^{-5} \mathrm{~mA} / \mathrm{cm}^{2}\right)$ & $\mathbf{V}_{\mathrm{oc}}(\mathbf{V})$ & FF & $\eta\left(10^{-5} \%\right)$ & $\mathbf{E}_{\mathrm{G}}(\mathrm{eV})$ \\
\hline RUB & - & - & - & - & 2.2 \\
\hline$\alpha-4 \mathrm{~T}$ & 7.3 & 1.8 & 0.35 & 5.0 & 2.6 \\
\hline TEN & 61 & 1.6 & 0.30 & 34 & 2.3 \\
\hline
\end{tabular}

solar cell with the same thickness [2]. Moreover, we stress that for PEL and $\alpha-4 \mathrm{~T}$, current/voltage characteristics in the device configuration of Fig. (1) were never reported, probably due to crystal vulnerability to traditional metallization processes.

Despite the high $V_{o c}$ of single crystal cells, the fill-factors $(F F)$ fall in the typical range of other bi-layer or bulk organic hetero-junctions. Variation of $F F$ can be related with the conformity of the interface contact between the crystal and the electrodes.

The optical absorbance spectra in Fig. (2) can help to explain the difference in electrical response of the materials used. Indeed, only a small fraction of the irradiated energy can be absorbed by PEL and $\alpha-4 T$, whereas a far more favorable spectral overlap is evidenced in the case of RUB and TEN. This observation accounts in part for the higher performance (in terms of $\eta$ ) of solar cells based on TEN and allows us to attribute to electron-hole recombination the reason for the absence of PV effect in RUB single crystal devices. In this respect, charge recombination can follow two different paths: radiative and non-radiative. The latter process is particularly detrimental in the case of solar cell devices because it transforms all the excitonic energy in thermal energy. Unfortunately, due to the intermolecular interaction, photoluminescence is often quenched in single crystalline phases, making non-radiative recombination a dominant event of the thermodynamics of the solar cell. This observation is substantiated by the work carried out by Pandey et al. [4], where the $\mathrm{PV}$ behavior of $\mathrm{RUB} / \mathrm{C}_{60}$ heterostructures is demonstrated to originate from the light absorption of only the $\mathrm{C}_{60}$ layer, whereas excitons in RUB do not undergo efficient charge transfer.

In order to try to correlate the experimentally extracted PV parameters with the electronic structure of the active layers, we report in the last column of Table $\mathbf{1}$ the optical absorption edge $\left(E_{G}\right)$ deduced from the graphs in Fig. (2). As can be seen, the difference $E_{G}-e V_{o c}$ varies from 0.7 to 1.1 $\mathrm{eV}$, this representing an estimate of the energy loss excited electrons undergo before charge collection at the cathode. Being all the studied systems single layer devices, as nicely demonstrated in Ref. [25] the loss in open-circuit voltage can be attributed to the non-radiative recombination of charges within the crystalline layer. It is evident that the rate constant for recombination of excitons is an intrinsic properties of the material. For RUB this is relatively high, preventing the measurement of a finite $V_{o c}$, while TEN exhibits the lowest one.

\section{CONCLUSIONS}

We measured for the first time the PV response of single crystalline devices based on RUB, PEL, $\alpha-4$ T, and TEN. This has been shown to be characterized by relatively high values of $V_{o c}$, reflecting the high energy gap of the organic semiconducting layer. A high rate constant of exciton recombination was suggested as the main process giving rise to a low power conversion efficiency of single-layer singlecrystalline devices. This effect is particularly evident in RUB single crystals, which does not give rise to a detectable photocurrent. Among the used materials, TEN revealed to be the most promising one for the fabrication of crystalline organic devices, since it exhibits the lowest rate constant of recombination. On the other hand, the low performance of RUB single layer devices in terms of $V_{o c}$ and photocurrent does not establishes the inadequacy of this materials for the fabrication of solar cells. Its well-known high carrier mobility can be exploited in devices with an heterojunction configuration, where excitons are generated in the acceptor phase.

\section{ACKNOWLEDGEMENTS}

This work was supported by Fondazione Cariplo (Grant n. 2007/5205).

\section{REFERENCES}

[1] Riede M, Mueller T, Tress W, Schueppel R, Leo K. Small molecule solar cells-status and perspectives. Nanotechnology 2008; 19: 424001.

[2] Tseng RJ, Chan R, Tung VC, Yang Y. Anisotropy in organic single-crystal photovoltaic characteristics. Adv Mater 2008; 20: 4358 .

[3] Brütting W. Physics of organic semiconductors. Wiley-VCH Weinheim, Germany 2005.

[4] Pandey AK, Nunzi J-M. Rurbene/fullerene heterostructures with half-gap electroluminescence threshold and large photovoltage. Adv Mater 2007; 19: 3613-7.

[5] Kotani M, Kakinuma K, Yoshimura M, et al. Charge carrier transport in high purity perylene single crystal studied by time-of-fligh measurements and through field effect transistor characteristics. Chem Phys 2006; 325: 160-9.

[6] Unni KNN, de Bettignies R, Dabos-Seignon S, Nunzi J-M. A nonvolatile memory element based on a quaterthiophene field-effect transistor. Mater Lett 2005; 59: 1165-8.

[7] Laudise RA, Kloc Ch, Simpkins PG, Siegrist T. Physical vapor growth of organic semiconductors. J Crystal Growth 1998; 187: 449-54.

[8] Campione M, Ruggerone R, Tavazzi S, Moret M. Growth and characterization of centimetre-sized molecular organic single crystals. J Mater Chem 2005; 15: 2437-43.

[9] Jurchescu OD, Meetsma A, Palstra TTM. Low-temperature structure of rubrene single crystals grown by vapor transport. Acta Crystallogr B 2006; 62: 330-4. 
[10] Huang L, Liao Q, Shi Q, Fu H, Ma J, Yao J. Rubrene microcrystals from solution routes: their crystallography, morphology, and optical properties. J Mater Chem 2010; 20: 159-66.

[11] Botoshansky M, Herbstein FH, Kapon M. Towards a complete description of a polymorphic crystal, the example of perylene Redetermination of the structures of the $(Z=2$ and 4) Polymorphs. Helv Chim Acta 2003; 86: 1113-28.

[12] Tanaka J. The electronic spectra of aromatic molecular crystals. II: the crystal structure and spectra of perylene. Bull Chem Soc Jpn 1963; 36: 1237-49.

[13] Siegrist T, Kloc C, Laudise RA, Katz HE, Haddon RC. Crystal growth, structure, and electronic band structure of $\alpha-4 \mathrm{~T}$ polymorphs. Adv Mater 1998; 10: 379-82.

[14] Holmes D, Kumaraswamy S, Matzger AJ, Vollhardt KPC. On the nature of nonplanarity in the [N]phenylenes. Chem Eur J 1999; 5: 3399-412.

[15] Campione M, Raimondo L, Sassella A. Hetero-epitaxy of $\alpha$ quaterthiophene on tetracene single crystals. J Phys Chem C 2009; 111: 19009-14.

[16] Tavazzi S, Silvestri L, Campione M, et al. Generalized ellipsometry and dielectric tensor of rubrene single crystals. J Appl Phys 2007; 102: 023107.

[17] Tavazzi S, Campione M, Laicini M, Raimondo L, Borghesi A, Spearman P. Measured davydov splitting in oligothiophene crystals. J Chem Phys 2006; 124: 194710.
[18] Tavazzi S, Raimondo L, Silvestri L, et al. Dielectric tensor of tetracene single crystal: the effect of anisotropy on polarized absorption and emission spectra. J Chem Phys 2008: 128, 154709.

[19] de Boer RWI, Morpurgo AF. Influence of surface traps on spacecharge limited current. Phys Rev B 2005; 72: 073207.

[20] Pesavento PV, Puntambekar KP, Frisbie CD, McKeen JC, Ruden PP. Film and contact resistance in pentacene thin-film transistors: dependence on film thickness, electrode geometry, and correlation with hole mobility. J Appl Phys 2006; 99: 094504.

[21] Lioubtchenko DV, Markov IA, Briantseva TA. GaAs surface modifications under Au evaporating flux. Appl Surf Sci 2003; 211: 33540.

[22] Horcas I, Fernández R, Gómez-Rodríguez JM, Colchero J, GómezHerrero J, Baro AM. WSXM: a software for scanning probe microscopy and a tool for nanotechnology. Rev Sci Instrum 2007; 78: 013705 .

[23] Campione M, Sassella A, Moret M, Braga D. Method of manufacturing electrical contacts on organic semiconductors. EP09425182.4, May 12, 2009.

[24] Somani PR, Somani SP, Umeno M. Toward organic thick film solar cells: Three dimensional bulk heterojunction organic thick film solar cell using fullerene single crystal nanorods. Appl Phys Lett 2007; 91: 173503 .

[25] Kirchartz T, Taretto K, Rau U. Efficiency limits of organic bulk heterojunction solar cells. J Phys Chem C 2009; 113: 17958-66.

(C) Campione et al.; Licensee Bentham Open.

This is an open access article licensed under the terms of the Creative Commons Attribution Non-Commercial License (http://creativecommons.org/licenses/by-nc/3.0/) which permits unrestricted, non-commercial use, distribution and reproduction in any medium, provided the work is properly cited. 